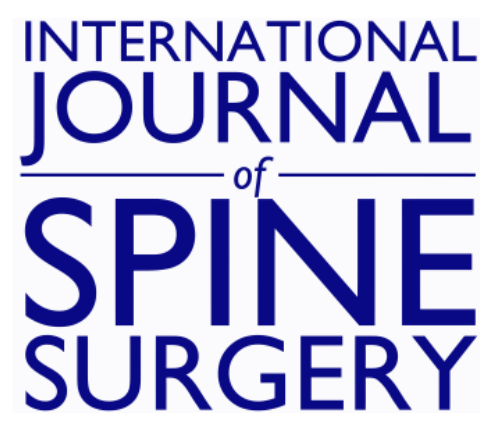

\title{
The surgical learning curve and accuracy of minimally invasive lumbar pedicle screw placement using CT based computer-assisted navigation plus continuous electromyography monitoring - a retrospective review of 627 screws in 150 patients
}

Martin James Wood and Jason McMillen

Int J Spine Surg 2014, 8 ()

doi: https://doi.org/10.14444/1027

http://ijssurgery.com/content/8/27

This information is current as of April 26, 2023.

Email Alerts Receive free email-alerts when new articles cite this article. Sign up at: http://ijssurgery.com/alerts 


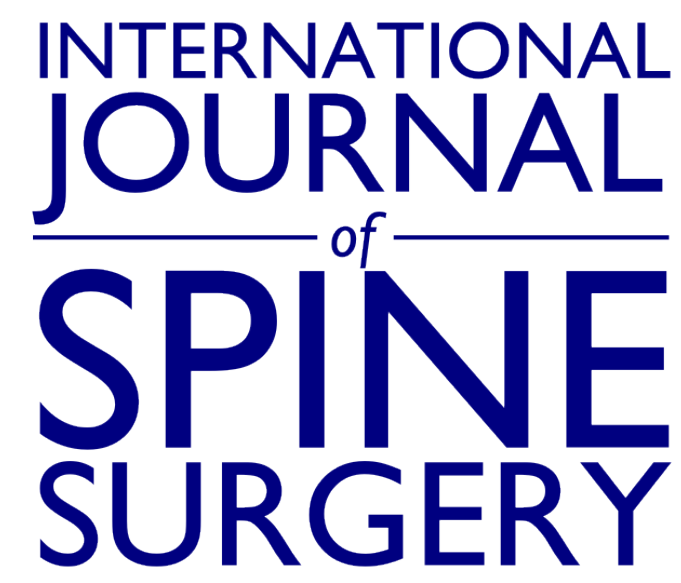

This article generously published free of charge by the International Society for the Advancement of Spine Surgery.

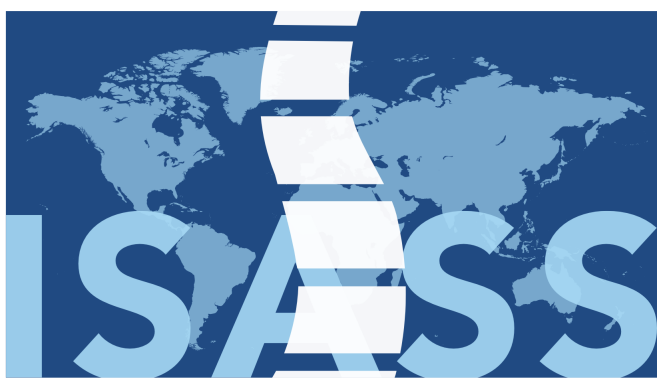

INTERNATIONAL SOCIETY for the ADVANCEMENT of SPINE SURGERY 


\section{The surgical learning curve and accuracy of minimally invasive lumbar pedicle screw placement using CT based computer-assisted navigation plus continuous electromyography monitoring - a retrospective review of 627 screws in 150 patients.}

Martin James Wood M.B.Ch.B, FRACS, Jason McMillen, MBBS

Mater Misericordiae Private Hospital, South Brisbane, Queensland, Australia

\section{Abstract}

\section{Objective}

This study retrospectively assessed the accuracy of placement of lumbar pedicle screws placed by a single surgeon using a minimally-invasive, intra-operative CT-based computer navigated technique in combination with continuous electromyography (EMG) monitoring. The rates of incorrectly positioned screws were reviewed in the context of the surgeon's experience and learning curve.

\section{Methods}

Data was retrospectively reviewed from all consecutive minimally invasive lumbar fusions performed by the primary author over a period of over 4 years from April 2008 until October 2012. All cases that had utilized computer-assisted intra-operative CT-based image guidance and continuous EMG monitoring to guide percutaneous pedicle screw placement were analysed for the rates of malposition of the pedicle screws. Pedicle screw malposition was defined as having occurred if the screw trajectory was adjusted intraoperatively due to positive EMG responses, or due to breach of the pedicle cortex by more than $2 \mathrm{~mm}$ on intraoperative CT imaging performed at the end of the instrumentation procedure. Further analysis of the data was undertaken to determine if the rates of malposition changed with the surgeon's experience with the technique. 


\section{Results}

Six hundred and twenty-seven pedicle screws were placed in one hundred and fifty patients. The overall rate of intraoperative malposition and subsequent adjustment of pedicle screw placement was 3.8\% (24 of 627 screws). Screw malposition was detected by intraoperative CT imaging. Warning of potential screw misplacement was provided by use of the EMG monitoring. With increased experience with the technique, rates of intraoperative pedicle screw malposition were found to decrease from $5.1 \%$ of screws in the first fifty patients, to $2.0 \%$ in the last 50 patients. Only one screw was suboptimally placed at the end of surgery, which did not result in a neurological deficit.

\section{Conclusion}

The use of CT-based computer-assisted navigation in combination with continuous EMG monitoring during percutaneous transpedicular screw placement results in very low rates of malposition and neural injury that compare favourably with previously reported rates. Pedicle screw placement accuracy continues to improve as the surgeon becomes more experienced with the technique.

keywords: minimally invasive spine surgery, lumbar fusion, Pedicle screw, Spinal navigation, learning curve Volume 8 Article 27 doi: 10.14444/1027

\section{Introduction}

The use of pedicle screw fixation of the lumbar spine to correct deformity and promote osseous fusion is a well established technique, commonly utilized as an adjunct to interbody arthrodesis procedures such as posterior lumbar interbody fusion (PLIF) and transforaminal lumbar interbody fusion (TLIF). Correct placement of the pedicle screw within the cortex of the pedicle is considered critical to avoid damage to adjacent neural structures, as well as to ensure optimal purchase within the bone. Failure to place pedicle screws correctly may result in neurological injury, reduce structural strength of the stabilising construct and increase rates of pseudarthrosis and instrumentation failure.

The classic free hand open techniques for placement of pedicle screws have relied on the use of vertebral anatomical landmarks and tactile feedback to facilitate correct screw positioning and avoid neural injury. Previous studies of the accuracy of this technique have demonstrated pedicle wall breach rates ranging from $5 \%$ to $31 \% .1,2,3,4,5,6$

Consequently, these techniques have been enhanced with the use of intraoperative fluoroscopy to guide trajectory and placement of the pedicle screws, using the intraoperative $\mathrm{C}$-arm to image the field in two planes. The development and widespread availability in recent years of computer-assisted navigation techniques have added further to the tools available to the surgeon to minimise the risk of screw malposition, with the additional benefit of reducing the radiation dose to the surgical team. These include both fluoroscopy- and computerised tomography (CT)-based navigation technologies.

Over the last decade, techniques to perform instrumentation of the lumbar spine through minimally invasive, percutaneous muscle-splitting approaches have been developed. These techniques are postulated to offer advantages over open spinal surgical techniques, 
including minimising damage to paraspinal soft tissues and musculature, preservation of midline ligamentous structures and lower rates of surgical complications such as blood loss. However, the minimally invasive spine surgeon sacrifices much of the tactile and visual feedback relied upon during open pedicle screw placement, as the smaller incisions and reduced soft tissue dissection inherent in these techniques limit visualisation and palpation of anatomical landmarks. Minimally invasive placement of pedicle screws is therefore much more dependent on intraoperative imaging such as fluoroscopy or real time computer-assisted image-guided navigation to reduce the risk of pedicle wall breach and screw malposition. Using these techniques, accuracy rates of pedicle screw placement comparing favourably with those of image guided open procedures have been reported, but much of the learning curve of adoption of these minimally invasive techniques relates to safe placement of spinal instrumentation.

A second technique that has been shown to improve further the accuracy of pedicle screw placement in both conventional open and minimally invasive spinal surgery is the use of intraoperative continuous electromyography (EMG) monitoring. In this technique, the instrumented pedicle is stimulated with a continuous current through an insulated pedicle access needle. When the pedicle wall has been breached, responses in the adjacent nerve root and related lower limb myotome may be recorded through percutaneous EMG electrodes in the lower limb. This will alert the surgeon to a pedicle wall breach and potential nerve root injury, and allow correction of the pedicle screw trajectory to avoid neural compromise. Use of EMG monitoring in both thoracic and lumbar pedicle screw instrumentation has previously been shown to be accurate in detecting pedicle wall breaches and potential neurological injury. ${ }^{7,8,9}$ Continuous lower limb EMG monitoring has been reported as a useful adjunct to computer-assisted navigation to improve pedicle screw accuracy by alerting the surgeon to potential pedicle wall breaches before they are detected using intra- or postoperative imaging. $8,10,11$

In this study we review a single surgeon's series of patients in whom percutaneous lumbar pedicle screws were placed using a computer-assisted intraoperative CT image-guided navigation technique, enhanced by the use of continuous lower limb EMG monitoring. Pedicle screw positioning was assessed using intraoperative CT scanning after placement of the instrumentation. The reported rates of intraoperative screw adjustment, resulting from positive EMG signals or pedicle breach detected using navigation or CT scanning at the end of the procedure, are reported, as well as rates of suboptimally positioned screws that were not adjusted. Accuracy rates were analysed in the context of the surgeon's experience with the technique and advancement along the learning curve.

\section{Methods}

\section{Patient population}

A total of 150 consecutive patients ( 83 female, 67 male) with a mean age of 58 years (range 21-88 years) underwent minimally invasive lumbar fusion surgery with percutaneous pedicle screws between April 2008 and October 2012. All patients except one had degenerative lumbar spinal conditions. All the pedicle screws were placed with the assistance of real-time computer-assisted image guidance using on-table 
intraoperative CT imaging acquisition. Continuous lower limb EMG monitoring was performed in all instances to assess for potential pedicle cortical breaches and nerve root injury. All cases were performed by one surgeon (MJW).

Patient data regarding intraoperative complications were prospectively recorded in a clinical database. Specifically, the incidence of screw adjustments resulting from either a positive intraoperative EMG signal, or malposition identified on intraoperative CT imaging, were noted. If a screw breached the pedicle cortical wall, the direction of breach (superior, lateral, inferior, or medial) was recorded, and it was noted if the breach was confirmed by a positive EMG signal. Any suboptimally positioned screws that the surgeon felt did not require repositioning were also recorded.

As surgeons' familiarity with a surgical technique is related to the number of cases rather than the dates at which they are performed, further analysis of the data was undertaken to determine if any detectable changes in the incidence of screw malposition and adjustment occurred with increasing surgical experience and case numbers.

\section{Operative technique}

In all patients a computer-assisted, optical image-guided system (Stealth Station, Medtronic, Minneapolis, MN, USA) with stimulus evoked electromyography (EMG) monitoring of nerve roots was used. All cases were performed under general anaesthesia. Muscle relaxants were not utilised, to facilitate continuous EMG monitoring. Intramuscular needle electrodes were place in the lower limb musculature corresponding to the nerve root myotomes of the levels to be instrumented. A reference electrode was placed into the external oblique muscle at the patient's flank, and a grounding return electrode placed on the buttock. Standard mechanical venous thromboembolism precautions (compression stockings and sequential pneumatic compressive devices) were applied to the lower limbs. The electrodes were connected to a monitoring computer (Neural Integrity Monitor [NIM], Medtronic). After placing the patient in the prone position on a radiolucent operating table, the image guidance system reference array was placed percutaneously into the posterior iliac crest and radiographic images obtained on which to base subsequent surgical navigation. In all patients an intraoperative threedimensional (3D) fluoroscopic system (O-arm, Medtronic) was used to acquire on-table images. These images were then used to guide placement of the pedicle screws and interbody implants with reference to the image guidance system, without further requirement for intraoperative imaging until the conclusion of the surgery.

\section{Implant placement technique}

By placing a "virtual" extension on the surgical probe of the navigation system, the correct skin incision, entry point and trajectory of each pedicle screw was planned so as to place the entry of the pedicle screw at the junction of the transverse process and the facet complex, avoiding the articular surface of the facet joint. A small skin incision was then made at the point corresponding to this trajectory. Using an insulated pedicle access needle that was visible to the image guidance system and connected to the EMG monitoring system (NIM) with a stimulus of $5 \mathrm{~mA}$, the pedicle was cannulated. If an audible alert signal was produced by the EMG monitoring system during pedicle cannulation, the trajectory of the pedicle access needle was modified, using the image 
guidance system as a further guide, until no EMG signal was detected, and the incident was recorded to the database. The pedicle access needle was then cannulated with a Kwire and the needle withdrawn. Serial dilators were placed over the K-wire to create a muscular tunnel to the pedicle screw entry point. The pedicle tap was placed, and stimulated with the EMG monitoring system to ensure that the larger diameter of the tap had not resulted in a pedicle breach. Subsequently the pedicle screw was placed. All pedicles were cannulated prior to performing the TLIF, as the distraction of the disc space at this phase of the procedure may have rendered the navigation system inaccurate and made subsequent pedicle cannulation potentially unsafe. It is difficult to perform the TLIF procedure if the pedicle screws with their extenders are in place on the same side from which the TLIF is to be performed. For a TLIF, we therefore inserted the pedicle screws on the contralateral side, but on the ipsilateral side, we cannulated the pedicle with the Kwire only and did not insert the ipsilateral screws until after the interbody cage was inserted. This allowed unimpeded insertion of muscular dilators and an unobstructed view down the operating tube. The decompression and interbody fusion was performed via a 20 $\mathrm{mm}$ cylindrical operating tube with an operating microscope. Rather than real-time fluoroscopy, the image guidance system was used to guide the placement of the operating tube onto the facet joint. Intradiscal distraction of the operated level was achieved with lordotic disc space spreaders and then maintained by insertion and fixation of a percutaneous rod through the pedicle screws on the side contralateral to the TLIF. This facilitated endplate preparation and insertion of the interbody cage with the disc space distracted. Only the ipsilateral nerve root was directly decompressed, with the TLIF performed from the side of the greatest radicular symptoms. Once the interbody fusion was completed, the ipsilateral screws could be placed over the K-wires using a Seldinger technique. After all the pedicle screws were inserted, the screws were compressed over the interbody cage to provide a degree of segmental lordosis and compression across the cage. For a PLIF procedure, the pedicle screws were inserted bilaterally at the beginning of the procedure, as the screw extenders do not obstruct insertion of the muscle dilators and operating tube. In all cases, after the interbody cages, pedicle screws and rods were positioned, the placement of the instrumentation was assessed intraoperatively by both surgeons using the 3D fluoroscopic system. This enabled immediate assessment of the placement of the pedicle screws in axial, sagittal, and coronal planes. A malpositioned screw was defined as one that had at least $2 \mathrm{~mm}$ of the screw diameter breaching beyond the cortex of the pedicle. If a screw required adjustment, the direction of the breach was recorded to the database, and the screw was revised during the same procedure. Once the $\mathrm{O}$-arm images were judged to be satisfactory, the pedicle screw caps could be tightened and the screw extenders removed.

As the patients were imaged with 3D fluoroscopy within the operating theatre suite after placement of the instrumentation, no postoperative CT scanning was required.

\section{Results}

A total of 627 pedicle screws were placed in 150 consecutive cases in a single institution over a period from April 2008 until October 2012. 139 patients underwent minimally invasive transforaminal lumbar interbody fusion (TLIF) with placement of a single anterior interbody cage through a unilateral approach, and five patients underwent posterior lumbar interbody fusion (PLIF) with placement of bilateral interbody cages. Six 
patients underwent posterolateral fixation (PLF) alone, without use of an interbody device. All cases underwent instrumentation across one or two lumbar levels. One patient had a plasmacytoma in the T12 vertebral body, and underwent a minimally invasive resection of the tumour, with subsequent fixation from T11 to L1. The procedures and levels are listed in Table 1.

Table 1. Surgical procedures.

\begin{tabular}{|c|c|c|c|}
\hline Procedure & Number of patients & Procedure and level & Number of patients \\
\hline \multirow[t]{3}{*}{ Single level TLIF } & 124 & TLIF - L3-L4 & 2 \\
\hline & & TLIF - L4-L5 & 60 \\
\hline & & TLIF - L5-S1 & 62 \\
\hline \multirow[t]{2}{*}{2 level TLIF } & 15 & TLIF - L3-L5 & 1 \\
\hline & & TLIF - L4-S1 & 14 \\
\hline \multirow[t]{2}{*}{ Single level PLIF } & 5 & PLIF - L4-L5 & 1 \\
\hline & & PLIF - L5-S1 & 4 \\
\hline \multirow[t]{4}{*}{ Posterolateral fixation } & 6 & Postero-lateral Fixation - T11-L1 & 1 \\
\hline & & Postero-lateral Fixation - L4-L5 & 2 \\
\hline & & Postero-lateral Fixation - L5-S1 & 1 \\
\hline & & Postero-lateral Fixation - L4-S1 & 2 \\
\hline Total cases & 150 & & 150 \\
\hline
\end{tabular}

We were able to assess the effect of both continuous EMG monitoring and computer assisted navigation on the rates of intraoperative adjustment of pedicle screws. A summary of the rates of pedicle screw malposition and intraoperative adjustment is found in Tables 2 and 3, including the effects on these rates of increased surgical experience with the technique.

\section{Intraoperative EMG guided corrections}

The use of continuous electromyography recording during pedicle cannulation and screw placement resulted in a positive EMG signal in 19 (3.0\%) of 627 screws, in 17 of 150 cases (Table 2). A positive signal suggests proximity of the pedicle access needle to an adjacent nerve root, and possible pedicle cortical breach. The stimulus amplitude of $5 \mathrm{~mA}$ leads to an incidence of positive EMG signals that is not always associated with breaches of the pedicle cortex, but may be close enough to the pedicle cortex that subsequent pedicle tapping and screw placement may cause a defect in the pedicle wall. Therefore in all cases with a positive signal the trajectory of the pedicle access needle was adjusted until no EMG signal was induced. 
Table 2. Complication learning curve - Pedicle access trajectory malposition and adjustment due to EMG monitoring.

\begin{tabular}{|c|c|c|c|c|}
\hline & $\begin{array}{l}\text { First } 50 \\
\text { April 2008-Mar } \\
2010\end{array}$ & $\begin{array}{l}\text { Second } 50 \\
\text { May } 2010 \text { - August } \\
2011\end{array}$ & $\begin{array}{l}\text { Third } 50 \\
\text { Sept } 2011 \text { - October } \\
2012\end{array}$ & Total \\
\hline Cases adjusted due to EMG & $\begin{array}{l}8 / 50 \text { cases } \\
=16 \%\end{array}$ & $\begin{array}{l}6 / 50 \text { cases } \\
=12 \%\end{array}$ & $\begin{array}{l}3 / 50 \text { cases } \\
=6 \%\end{array}$ & $\begin{array}{l}17 / 150 \text { cases } \\
=11.3 \%\end{array}$ \\
\hline $\begin{array}{l}\text { Pedicle access trajectories adjusted due to } \\
\text { EMG }\end{array}$ & $\begin{array}{l}9 / 216 \text { screws } \\
=4.2 \%\end{array}$ & $\begin{array}{l}7 / 209 \text { screws } \\
=3.3 \%\end{array}$ & $\begin{array}{l}3 / 202 \text { screws } \\
=1.5 \%\end{array}$ & $\begin{array}{l}19 / 627 \\
\text { screws } \\
=3.0 \%\end{array}$ \\
\hline
\end{tabular}

\section{Intraoperative CT guided corrections}

Pedicle screw position was assessed with intraoperative CT imaging using the $\mathrm{O}$-arm device prior to completion of each procedure. Using the criteria initially described by Gertzbein and Robbins, ${ }^{12}$ any pedicle screw breaching the cortex of the pedicle by more than $2 \mathrm{~mm}$, or that was felt to have a risk of encroachment on neural structures, was repositioned. Despite the use of intraoperative image-guidance and continuous EMG monitoring to assist with correct placement of screws within the pedicle, 8 of 627 screws $(1.3 \%)$ were judged to be positioned sub-optimally when reviewed on intraoperative CT imaging at the end of the procedure, as shown in Table 3 . The most common direction of breach was laterally, as shown in Table 4.

Table 3. Complication learning curve - Screw malposition and adjustment due to intraoperative CT.

\begin{tabular}{|c|c|c|c|c|}
\hline & $\begin{array}{l}\text { First } 50 \\
\text { April } 2008 \text { - Mar } \\
2010\end{array}$ & $\begin{array}{l}\text { Second } 50 \\
\text { May } 2010 \text { - August } \\
2011\end{array}$ & $\begin{array}{l}\text { Third } 50 \\
\text { Sept } 2011 \text { - October } \\
2012\end{array}$ & Total \\
\hline Screws adjusted due to $\mathrm{CT}$ & $\begin{array}{l}4 / 216 \text { screws } \\
=1.9 \%\end{array}$ & $\begin{array}{l}3 / 209 \text { screws } \\
=1.4 \%\end{array}$ & $\begin{array}{l}1 / 202 \text { screws } \\
=0.5 \%\end{array}$ & $\begin{array}{l}8 / 627 \text { screws } \\
=1.3 \%\end{array}$ \\
\hline $\begin{array}{l}\text { TOTAL adjusted trajectories + } \\
\text { screws } \\
(\text { EMG }+ \text { CT })\end{array}$ & $\begin{array}{l}11 / 216 \text { screws } \\
=5.1 \%\end{array}$ & $\begin{array}{l}9 / 209 \text { screws } \\
=4.3 \%\end{array}$ & $\begin{array}{l}4 / 202 \text { screws } \\
=2.0 \%\end{array}$ & $\begin{array}{l}24 / 627 \\
\text { screws } \\
=3.8 \%\end{array}$ \\
\hline
\end{tabular}

Note: Some adjusted screws were malpositioned on intraoperative CT, as well as EMG signal positive.

Table 4. Direction of pedicle breach of malpositioned pedicle screws.

\begin{tabular}{|l|l|l|l|l|}
\hline & Lateral breach & Superior breach & Inferior breach & Medial breach \\
\hline EMG positive & 1 & 2 & 0 & 0 \\
\hline EMG Negative & 3 & 0 & 1 & 1 \\
\hline
\end{tabular}

One screw breaching the pedicle wall laterally in a two-level TLIF was judged to be safe to remain unadjusted, as the breach was $<2 \mathrm{~mm}$ in diameter and there was no threat to neural structures. 
Five pedicle breaches were detected by $\mathrm{CT}$ in the absence of a positive EMG signal. Two of these screws had breached inferiorly or medially to the pedicle, where the exiting nerve root is most intimately related to the pedicle, but each by less than $2 \mathrm{~mm}$. No neurological symptoms resulted. The most common direction of pedicle breach in the absence of a positive EMG signal was lateral, reflecting the fact that a lateral breach is unlikely to threaten a nerve root. However, three pedicle breaches in the lateral and superior directions were highlighted by both a positive EMG signal and CT evidence of cortical breach. It is unclear in these cases whether the EMG signals were falsely positive, or whether neural compromise can still occur in these instances.

\section{Effect of surgeon experience}

The incidence of screw malposition and subsequent intraoperative adjustment was analysed in the context of increasing familiarity and expertise of the surgeon with the technique. The rates of pedicle cannulation trajectory adjustment per surgical case fell from an initial incidence of 8 cases requiring intraoperative adjustment in the first 50 cases $(16 \%)$ to a rate of 3 of the final 50 cases $(6.0 \%)$. Similarly, the total rates of malpositioned pedicle cannulation or screw placement requiring adjustment related to the total number of screws placed fell from 11 of 216 screws $(5.1 \%)$ in the first 50 cases, to 9 of 209 screws $(4.3 \%)$ in the second 50 cases, and 4 of 202 screws $(2.0 \%)$ in the final 50 cases. The rates of screw adjustment subsequent to both monitoring modalities, EMG and intraoperative $\mathrm{CT}$, both showed reducing rates with increasing surgical expertise.

Trajectories that were adjusted due to positive EMG signals decreased from 9 of 216 screws placed (4.2\%) in the first 50 cases to 3 of 202 screws $(1.5 \%)$ in the last 50 cases performed over the final 14 months of the study. Pedicle screws that were malpositioned on end-of-procedure intraoperative CT imaging fell from 4 of 216 screws (1.9\%) to 1 of 202 screws (0.5\%). A summary of these results appears in Figure 1. 


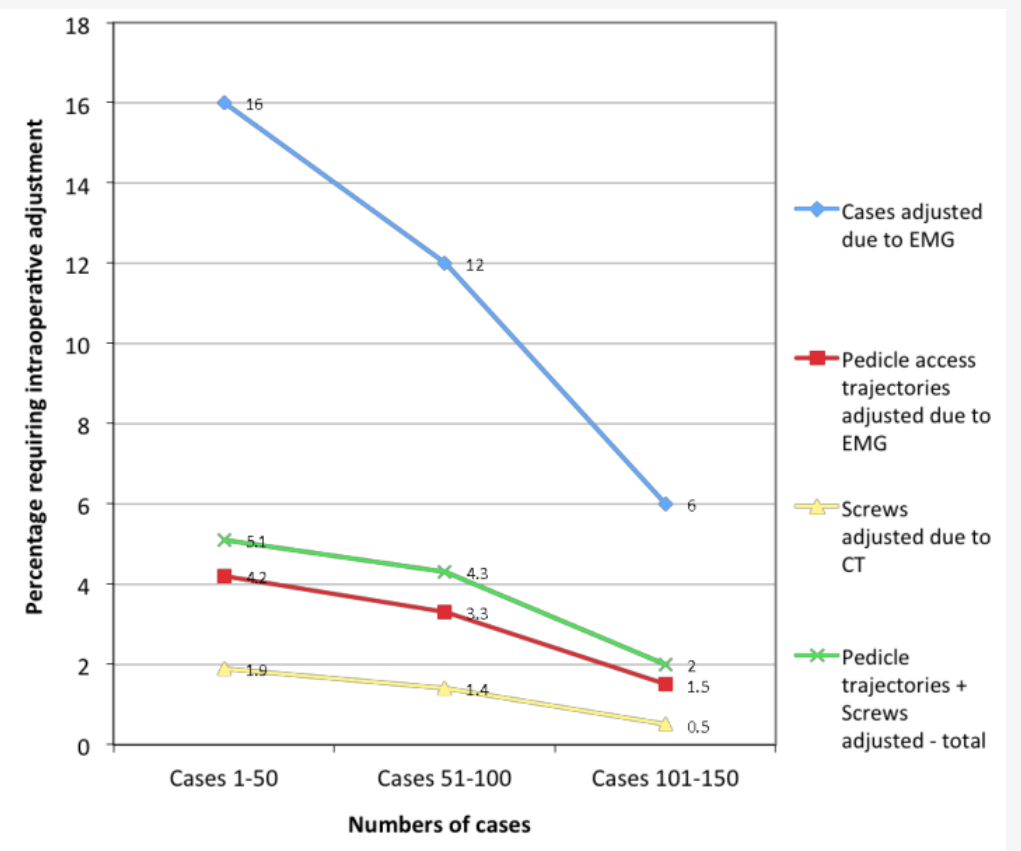

Fig. 1. Pedicle screw malposition and adjustment learning curve.

\section{Complications}

This technique allowed us to radiologically assess the position of the pedicle screws intraoperatively, and to avoid having to return to the operating theatre for a second procedure to adjust any unacceptably malpositioned screws. One pedicle screw in this series was judged to be positioned suboptimally with a breach in the lateral pedicle wall, but it did not induce an EMG signal, and post operatively was asymptomatic.

None of the cases in this case series suffered a new neurological deficit directly attributable to placement of the pedicle screws. One patient who underwent L5-S1 TLIF for spondylolisthesis experienced transient L5 radicular pain, despite having negative EMG signals throughout the procedure and $\mathrm{O}$-arm imaging confirming pedicle screw placement within the pedicles. A second patient who had undergone L3-4 TLIF for spondylolisthesis and stenosis developed a new footdrop. This patient also had normal intraoperative EMG monitoring, and intraoperative CT imaging confirming correct pedicle screw placement. Four patients undergoing TLIF procedures suffered from unintended durotomies. None of these patients required further intervention or suffered adverse sequelae from this complication.

\section{Discussion}

Since the initial development of transpedicular screw fixation techniques in the 1960s, techniques have evolved dramatically. Malposition of pedicle screws has long been recognized as a potential complication of these procedures, and can be associated with neural injury or irritation, CSF leak, pseudarthrosis, and instrumentation failure. Rates of screw malposition using conventional open techniques of placement have been reported as low as $5 \%$ and as high as $21-40 \%$ both radiologically and in cadaver studies. ${ }^{1,4,13}$ To improve accuracy of screw positioning, a number of techniques can be utilized such as 
laminectomy, pedicle palpation, plain radiography, intraoperative fluoroscopy, computerassisted image guidance, and intraoperative electromyographic monitoring. Even computer-assisted navigation has a number of variant methods, including guidance based on fluoroscopic images, fluoroscopy/CT image fusion, and intraoperative on-table CT imaging. All of these techniques have reported advantages and shortcomings, and most have lead to decreased rates of pedicle screw malposition and neurological injury in open screw insertion techniques. ${ }^{3,5,6,14,15,16}$ Despite this, the most common technique for placement of pedicle screws remains the open freehand technique, utilizing visible anatomical landmarks supplemented by intraoperative biplanar fluoroscopy. A confounding factor in gaining an accurate assessment of the rates of pedicle screw misplacement is the wide variability in surgical technique as relates to imaging practices. Some surgeons do not undertake any routine postoperative imaging (usually leading to an assumption of a screw misplacement rate of zero), some undertake plain radiographs alone, while others use CT scanning, which is significantly more sensitive to minor screw malposition.

The development of minimally invasive techniques of lumbar fusion has offered a number of potential advantages to the patient over standard open surgical techniques. Minimally invasive spinal surgery is associated with decreased blood loss and lower complication rates than open fusion procedures,${ }^{17}$ reduced injury to paraspinous soft tissues including the important long extensors and stabilising muscles of the spine, less postoperative pain, and early mobilization and discharge from hospital. ${ }^{10,18,19}$ Benefits to the surgical team include comparable operative time, and potentially reduced radiation exposure for the surgical team. ${ }^{11,20,21}$ Additionally, minimally invasive techniques avoid the need for lateral retraction of the paraspinal muscles to afford easier access to an entry point lateral to the facet joint than open techniques, facilitating easier avoidance of violation of the facet joints at levels adjacent to the level of fusion.

However, minimally invasive techniques for placement of pedicle screws present a number of new challenges when compared to open methods. These may lead to reluctance by some surgeons to adopt these techniques. Minimally invasive spinal surgical approaches preclude the use of direct visualization of anatomical landmarks as a guide to placement of pedicle screws, and therefore a freehand technique is very difficult and unsafe without radiological guidance. Surgeons performing percutaneous pedicle cannulation must therefore utilize intraoperative image guidance with either real-time fluoroscopy or computer-assisted navigation. Use of intraoperative fluoroscopy requires repeated exposure of both the surgical team and the patient to ionizing radiation, together with disadvantages in terms of requiring the maintenance of a fluoroscopic arm within the surgical field and presence of the radiographer throughout the procedure. Surgeons used to inserting pedicle screws with a conventional open freehand technique may be unwilling to adopt a new technique that is perceived to be longer and more difficult than that which they have been utilizing.

Intraoperative computer-assisted navigation with 2D fluoroscopy can reduce the exposure of the patient and surgical team to ionizing radiation. The only imaging required is conducted at the commencement of the procedure, with the reference array affixed to the patient. As long as the reference array remains stable in its position relative to the operative site, the accuracy of the navigation system remains uncompromised, and further 
imaging is only required at the completion of the case. However, issues with accuracy of two-dimensional imaging and assessment of pedicle screw position ${ }^{22}$ may lead to pedicle wall breaches that remain undetected until postoperative CT imaging is complete. The use of navigation technologies that utilise CT-based imaging mitigate this potential inaccuracy by enabling the surgeon to assess the planned screw trajectory in three dimensions. Pedicle wall breaches that are more difficult to detect with $2 \mathrm{D}$ fluoroscopic imaging such as lateral and especially medial violations may be avoided with this technique. The merging of preoperative supine $\mathrm{CT}$ images with the prone intraoperative fluoroscopic images is one technique to achieve CT images for navigation (not requiring an intraoperative $\mathrm{CT}$ or 3D fluoroscopic system), but may be subject to inaccuracies resulting from the differences in patient positioning. This may be exacerbated if the patient has a dynamic instability such as a spondylolisthesis.

Further improvements in the reliability of intraoperative navigation and placement of pedicle screws can be achieved with the use of computer-assisted navigation based on images obtained with an intraoperative CT system, of which the $\mathrm{O}$-arm is one example. High-resolution CT images of the spine in relation to a reference array are obtained once the patient is positioned on the operating table, negating any inaccuracies resulting from changes in position. Whilst this potentially exposes the patient to a greater dose of radiation than they might experience with biplanar fluoroscopy, the radiation dose to the surgical team can be reduced to nil, as they can leave the operating room as the images are being acquired. Whole body radiation exposure for the patient from high definition three dimensional O-arm cone-beam CT imaging has been shown to be comparable to, or less than the dose received from a 64 slice high definition diagnostic CT $(4.53 \mathrm{mSV}$ for O-arm imaging compared to $6.31 \mathrm{mSv}$ for 64 slice CT). ${ }^{23}$ The availability of threedimensional images greatly enhances the accuracy of tracking of navigated instruments and implants, and offers superior imaging quality to that obtained with fluoroscopy. Computer-assisted navigation based on three-dimensional imaging has been reported to lead to lower rates of malposition of pedicle screws when compared to 2D fluoroscopybased image guidance and to techniques that merge $2 \mathrm{D}$ fluoroscopy with preoperative CT. $6,21,24$ No further imaging is required during the procedure until after the instrumentation has been positioned, when it is our practice to obtain a second $\mathrm{O}$-arm image series to confirm correct placement of the pedicle screws. This second intraoperative CT affords the opportunity to correct any malpositioned screws and avoid a return to theatre, and obviates the need for a postoperative CT scan. A significant disadvantage of the O-arm system and a factor limiting its more widespread adoption is its cost. However Sanborn et al ${ }^{25}$ have determined that intraoperative use of the O-arm is a less costly method to confirm pedicle screw position than the use of intraoperative fluoroscopy and postoperative CT scanning. We suggest that a further reason for the slow adoption of CT imaging based computer-assisted navigation in spinal surgery is the learning curve associated with its initial use, and the reluctance of many surgeons to embark upon a period of learning new techniques. 


\section{Radiological assessment of accuracy of pedicle screw placement}

It is recognized that pedicle wall breaches, especially medial breaches which are most likely to be associated with neural encroachment due to the close relationship of the nerve root to the medial and inferomedial aspects of the pedicle, are less likely to be appreciated with plain radiography than with CT imaging. ${ }^{1,22,26} \mathrm{We}$ assessed our pedicle screw placement based on the system initially described by Gertzbein and Robbins, ${ }^{12,16}$ who considered that there is a margin of $4 \mathrm{~mm}$ adjacent to the pedicle that can be violated safely without impinging on the spinal cord or cauda equina. With the use of the intraoperative CT system, radiological assessment of screw position in three anatomical planes could be performed immediately after placement of the pedicle screws, before the patient was removed from the operating theatre (Figure 2). This affords the opportunity to revise any screws judged to be misplaced without the cost and inconvenience of a second operation. This is a significant advantage over the use of conventional postoperative CT imaging. Our reoperation rate in this series was zero.

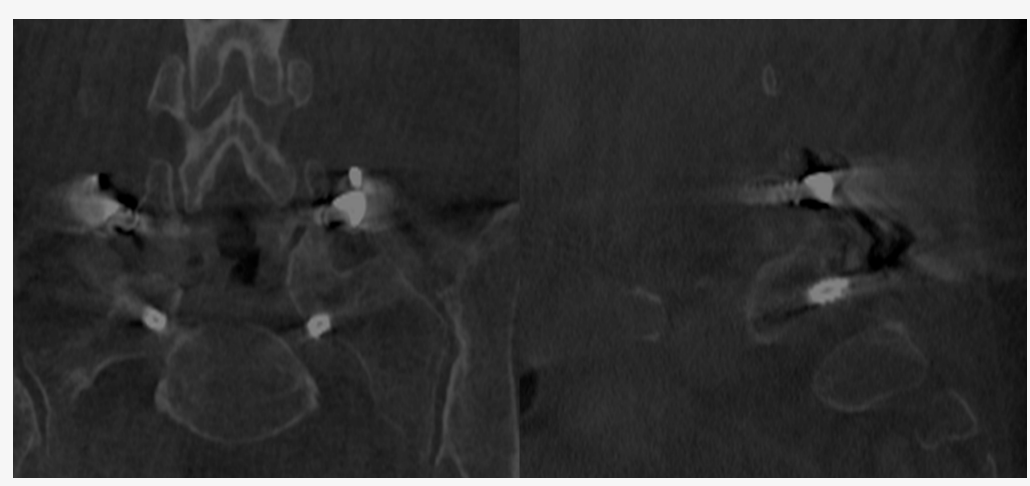

Fig. 2. Intraoperative CT imaging after L5-S1 TLIF, demonstrating an inferior breach of the cortex of the $\mathrm{S} 1$ pedicle by $<2 \mathrm{~mm}$.

Our overall rate of malpositioned screws detected by radiological assessment was $1.3 \%$. This compares favourably with the best rates reported using open freehand techniques. ${ }^{2}$ Studies of navigated techniques of pedicle screw insertion report rates of pedicle violation from $3 \%$ to $6 \%,{ }^{3,5,14,16,27}$ utilizing different modalities of imaging (2D fluoroscopy, 3D CT) in both open and minimally invasive surgical techniques.

The severity of violations of the pedicle wall has been reported to be greater in conventional freehand pedicle screw placement compared to screws placed with computer-assisted navigation. Ughwanogho et $\mathrm{al}^{16}$ reported that a non-navigated screw was more likely to require intraoperative removal and repositioning than a navigated screw. Additionally screws judged to be unsafe were less likely to be inserted with navigation, and medial breaches with a higher risk of neurological injury were more likely to occur with non-navigated screws. This increased risk of medial pedicle wall breach with freehand screws was confirmed by Gelalis et al, ${ }^{2}$ who also noted that screws placed with CT image guidance were more likely to breach in the less hazardous lateral direction. Our study confirms this finding, with $50 \%$ of the pedicle wall breaches detected by intraoperative CT occurring laterally. None of the pedicle wall violations in this study resulted in a neurological deficit. 


\section{Intraoperative electromyography monitoring during pedicle screw placement}

The use of intraoperative continuous EMG monitoring as an adjunct to both open and minimally invasive surgery to place pedicle screws has been described previously. $., 9,11,27,29,30$ The development of commercially available EMG monitoring units that are easy to use without specialist neurophysiologists has brought this technique into more widespread use. Continuous intraoperative lower limb EMG monitoring can now be performed easily with minimal increase in theatre time, and a minimal increase in cost.

In order to perform continuous EMG monitoring the use of muscle relaxants should be avoided. This can be problematic in open spinal fusion procedures, where the lack of muscle relaxation can make lateral retraction of the paraspinal musculature difficult. In minimally invasive spinal fusions, the lack of muscle relaxation is not problematic, as there is minimal muscle retraction and a more direct trajectory of the operating tubes and instruments to the pedicle entry point.

In this series of cases utilizing intraoperative continuous lower limb EMG monitoring to supplement CT image-based computer-assisted minimally invasive percutaneous pedicle screw placement, $3.0 \%$ of initial pedicle trajectories with the pedicle access needle were adjusted due to positive EMG signals. It should be noted that the EMG monitoring system does not stimulate pedicle screws after they are placed. Rather, the EMG stimulus current is passed through a continuously active pedicle access needle, which has an insulated shaft and a metallic tip. This prevents passage of the current through the paraspinous soft tissues and false positive stimulation of nearby nerve roots. The tip of the active pedicle access needle is advanced into the pedicle using image guidance. An incorrect trajectory resulting in threatened or actual pedicle wall violation results in stimulation of the adjacent nerve root, associated with myoclonic lower limb twitching and an auditory warning signal. This allows adjustment of the trajectory of the pedicle access needle before any nerve injury has occurred. Once the pedicle access needle has been advanced through the pedicle without an EMG signal, it is cannulated with a K-wire, and the needle is withdrawn. Muscle dilators are then passed over the K-wire, the last of which is insulated. The navigated pedicle tap is passed over the K-wire, and the tap can be stimulated without the current passing through the soft tissues due to the insulated sleeve. After the pedicle screw has been placed, it can not be stimulated as the EMG stimulus current would pass through the metallic screw extender to the surrounding soft tissues.

There is no absolute value for a positive stimulation signal indicating screw misplacement - Raynor et al suggest that low threshold values are more likely to correlate with pedicle wall defects and possible contact with neural tissues. ${ }^{30}$ Conversely, high stimulation values will potentially stimulate lower limb nerves even through an intact pedicle cortical wall. With the Medtronic NIM system utilised in these cases, we found that a stimulation threshold of $5 \mathrm{~mA}$ was adequate to signal potential pedicle breaches without too high a rate of false positive signals. The experience of the primary surgeon in this series (MJW) with higher stimulation thresholds is discussed elsewhere. ${ }^{11}$ It is unclear how many of the positive EMG signals obtained in the cases described here were false positive results. Postulated factors influencing this include significant osteopenia or high sensitivity of a 
severely compressed nerve root. It should be noted that in all of our experience with the use of EMG, we have never seen a false negative response (ie. a silent EMG system that results in a neurological injury).

The close relationship of the nerve root to the inferior and medial aspect of the pedicle means that pedicle wall breaches in this direction are more likely to cause neurological injury than superior and lateral breaches. It follows that EMG monitoring is more likely to warn about medial and inferior breaches than lateral breaches, which may be "silent." This was confirmed by Bindal et al, who reported that EMG monitoring prevented any medial violations in their series, and the only pedicle wall breaches they reported were in the lateral wall. ${ }^{8}$ This was despite their use of a less accurate form of guidance in the form of intraoperative fluoroscopy. In their series, $76 \%$ of pedicle screw trajectories were adjusted as a result of positive EMG signals, a considerably higher rate than reported in our series, and reflecting both the utility of EMG monitoring to enhance safety of pedicle screw placement when used in combination with a less accurate method of image guidance, and the greater accuracy of CT image-based navigation in planning initial screw trajectories in our series.

In our series, continuous EMG monitoring was responsible for the adjustment of 19 pedicle screw trajectories in 17 cases. The addition of EMG monitoring to computerassisted navigation to enhance the accuracy of pedicle screw placement potentially reduced the rate of sub-optimally placed screws from $4.3 \%$ to $1.3 \%$, if one assumes a false-positive signal rate of zero. This improvement in accuracy occurs prior to the insertion of the screws, and prevents potential neurological injury that might occur intraoperatively as a result of a screw compromising a nerve root. The measured low rate of actual pedicle screw misplacement (1.3\%) detected by intraoperative CT is a result of the EMG monitoring preventing most pedicle cannulation problems before the screws are inserted, together with the high accuracy of using CT images for intraoperative navigation. ${ }^{24}$ We suggest that the role of continuous EMG monitoring is an adjunct to the use of image guidance, providing additional warning of potential neurological compromise that might not be appreciated using navigation alone, and allowing early avoidance of potential pedicle breaches by the screws, which have a larger diameter than the pedicle access needle. EMG monitoring may also act as a fail-safe in any case of loss of navigational accuracy, which may occur for example in very obese or osteopaenic patients in whom immobile bony purchase of the percutaneous reference frame in the iliac crest may be difficult to achieve. Navigational inaccuracy may not be appreciated immediately during surgery, and the addition of electrophysiological warning of potential neural compromise can give early warning to the surgeon of a loss of navigational accuracy which might not otherwise be noted until after screws have been implanted and end of procedure imaging is performed.

Not all pedicle wall violations were detected by EMG monitoring in our series. Intraoperative $\mathrm{CT}$ imaging after placement of the pedicle screws detected a total of five undetected pedicle wall breaches. As well as three "silent" lateral pedicle wall breaches, one pedicle was breached inferiorly and one breached medially. False negative results have been reported previously with EMG monitoring of pedicle screw placement, ${ }^{7}$ which reinforces our proposition that no single modality is infallible and none should be relied upon solely to guide placement of pedicle screws. Despite the utility of EMG monitoring 
in reducing the incidence of misplacement of screws in our series, the two screws that were positioned inferior and medial to the pedicle had potential to cause neurological injury, which was not detected by EMG monitoring. It should be noted though that these breaches were minor (ie. $2 \mathrm{~mm}$ or less of exposed screw), so it is our contention that the probability of significant neural injury from these pedicle screws was low.

\section{The surgical learning curve}

The existence of a learning curve in any surgical technique is well recognized, and may be reflected in an association of greater surgical experience with improvements in patient outcomes. Even early reports of pedicle screw data postulated improvements in the accuracy of pedicle screw positioning with increased familiarity with the technique. ${ }^{12}$ Our series of 150 consecutive computer-assisted minimally invasive pedicle screw cases over more than four years were all performed by one surgeon, in a single institution, using the same surgical technique. This therefore affords us the opportunity to measure the effect of the learning curve on a single outcome measure, namely the accuracy of pedicle screw placement.

Our series clearly demonstrates that the accuracy of pedicle screw placement improves as the surgeon becomes familiar with the technique. The number of cases requiring intraoperative screw trajectory and screw adjustment falls from $16 \%$ of cases in the first 50 cases performed, to less than half that rate in the final 50 cases. Similar rates of improvement are noted when analysing the proportion of screws requiring adjustment, due to potential screw malposition detected with either positive EMG signals, misplacement apparent on intraoperative CT scanning, or both. These data indicate that the initial complication rates and difficulties encountered with a novel surgical technique may not persist, and that a more accurate assessment of the true potential long term results of a surgical intervention may only become evident after an initial familiarization period whilst the surgeon climbs the learning curve.

Some surgeons may be reluctant to adopt minimally invasive techniques because of perceived or real early high complication rates and difficulties with the technique, including the lack of familiar landmarks and tactile and visual feedback during the procedures. Similar unfamiliarity with computer-assisted spinal navigation methods may have led to slow adoption of the techniques. It is certainly understandable that a surgeon may see little advantage to changing a technique that has served them well in the past to a surgical technique that is foreign to their previous experience, requires the use of new and expensive technologies, and that they may perceive as offering few advantages in time or initial outcomes. However, there is now increasing data that minimally invasive surgical fusion techniques can offer some outcomes superior to traditional open techniques, and that the use of computer-assisted spinal navigation can decrease the malposition and complication rates of pedicle screw fixation. We recognize that there is a learning curve associated with any new technique. Our data supports the proposition that surgeons that persist with these new techniques and work through the difficult initial part of their learning curve may start to experience improved outcomes when utilizing these techniques. As surgeons become more experienced in the techniques, the reported rates of complications may continue to improve below those reported in previous literature. 


\section{Radiation exposure}

One concern that can be raised with the use of intraoperative CT imaging with the O-arm device is the increased ionizing radiation exposure when compared to 2D fluoroscopy. The radiation exposure resulting from fluoroscopy screening techniques used with pedicle screw placement varies widely, as surgeons may take images after or during each screw, or only at the end of the insertion of all of the hardware. Fluoroscopic guidance of percutaneous pedicle screws necessitates the acquisition of multiple images as the Kwires are inserted into the pedicles, followed by the cannulated taps and screws. This radiation exposure is experienced by both the patient and the surgical team.

The use of the intraoperative CT results in essentially no radiation exposure to the operating theatre staff or surgeon, as the acquisition of the initial images for navigation occurs with the staff out of the room. ${ }^{20}$ Similarly, all the theatre staff leave the room for the final confirmation imaging. Whilst the patient may experience a higher level of radiation exposure than a fluoroscopy-based technique ${ }^{23}$, this is not a repeated exposure, and has distinct implications for improved navigational accuracy with 3D image guidance techniques. Computer-assisted navigation techniques have been shown to be able to reduce total radiation dose to the surgical team. ${ }^{11,21}$ Total radiation exposure for the patient from both the initial O-arm images for navigation as well as the final on-table Oarm images to confirm screw placement is equivalent to or less than two 64 slice $\mathrm{CT}$ scans, and obviates the need for a post operative CT scan. ${ }^{23}$ Whilst cone-beam CT imaging has some disadvantages compared to standard diagnostic CT scans, such as inaccurate assessment of tissue density, we feel that it is adequate for its use in this situation to define three dimensional bony anatomy and differentiate this from surrounding soft tissues with sub-millimetric accuracy. Additionally, in our experience, the use of the $\mathrm{O}$-arm adds very little additional time to the operation. The average duration of surgery in our series was 172 minutes (range 107-287 minutes).

The future: The use of operative robotic assistance has been suggested as a technique to improve pedicle screw placement accuracy as well as reduce radiation exposure. The early results are conflicting, with some reports ${ }^{31}$ of reduced radiation dose and improved accuracy, but other studies reporting worse screw positioning with no improvement in radiation exposure. ${ }^{32,33}$ Robotic guidance for pedicle screw placement is a new and developing technology, and further developments will surely be forthcoming in the future.

\section{Conclusion}

Our technique of minimally invasive pedicle screw placement guided by 3D computerassisted navigation based on intraoperative CT images and supplemented by intraoperative continuous EMG monitoring results in very low rates of pedicle screw misplacement and minimizes the risk of neurological injury. The addition of intraoperative CT confirmation of pedicle screw position at the end of the procedure prevents reoperation and reduces complications further. The use of intraoperative $\mathrm{CT}$ and navigation adds little time to the procedure, eliminates radiation exposure to the surgical team, and is cost effective. Accuracy improves as the surgeon becomes more familiar with the technique, demonstrating the effect of the learning curve. 


\section{References}

1. Amato V, Giannachi L, Irace C, Corona C. Accuracy of pedicle screw placement in the lumbosacral spine using conventional technique: computed tomography postoperative assessment in 102 consecutive patients. Journal of Neurosurgery Spine 12:306-313, 2010.

2. Gelalis IG, Paschos NK, Pakos EE, Politis AN, Arnaoutoglou CM, Karageorgos AC, Ploumis A, Xenakis TA. Accuracy of pedicle screw placement: a systematic review of prospective in vivo studies comparing free hand, fluoroscopy guidance and navigation techniques. European Spine Journal 21:247-255, 2012.

3. Laine T, Lund T, Ylikoski M, Lohikoski J, Schlenzka D. Accuracy of pedicle screw insertion with and without computer assistance: a randomised controlled clinical study in 100 consecutive patients. European Spine Journal 9:235-240, June 2000.

4. Laine T, Mäkitalo K, Schlenzka D, Tallroth K, Poussa M, Alho A. Accuracy of pedicle screw insertion: a prospective CT study in 30 low back patients. European Spine Journal 6:402-405, 1997.

5. Shin BJ, James AR, Njoku IU, Härtl R. Pedicle screw navigation: a systematic review and meta-analysis of perforation risk for computer-navigated versus freehand insertion. Journal of Neurosurgery - Spine 17(2):113-22, August 2012.

6. Tian N, Huang Q, Zhou P, Zhou Y, Wu R, Lou Y, Xu H. Pedicle screw insertion accuracy with different assisted methods: a systematic review and meta-analysis of comparative studies. European Spine Journal 20:846-859, 2011.

7. Moroney JM, Holladay FP. Continuous intraoperative electromyographic recording during spinal surgery. Journal of Neurosurgery. 82(3):401-5, March 1995.

8. Bindal RK, Ghosh S. Intraoperative electromyography monitoring in minimally invasive transforaminal lumbar interbody fusion. Journal of Neurosurgery - Spine 6:126-132, 2007.

9. Raynor BL, Lenke LG, Kim Y, Hanson DS, Wilson-Holden TJ, Bridwell KH, Padberg AM. Can Triggered Electromyograph Thresholds Predict Safe Thoracic Pedicle Screw Placement? Spine 27(18):2030-2035, September 2002.

10. Tsahtsarlis A, Wood M.Minimally invasive transforaminal lumbar interbody fusion and spondylolisthesis. Journal of Clinical Neuroscience 19(6):858-61, June 2012.

11. Wood MJ, Mannion RJ. Improving accuracy and reducing radiation exposure in minimally invasive lumbar interbody fusion. Journal of Neurosurgery - Spine 12:533-539, 2010.

12. Gertzbein SD, Robbins SE. Accuracy of pedicle screw placement in vivo. Spine 15(1):11-14, January 1990.

13. Blasius S. Accuracy of pedicle screw placement in lumbar vertebrae. Spine 21(11):1320-4, June 1996.

14. Lim MR, Girardi FP, Yoon SC, Huang RC, Cammisa FP Jr. Accuracy of Computerized Frameless Stereotactic Image-Guided Pedicle Screw Placement into Previously Fused Lumbar Spines. Spine 30(15):1793-1798, August 2005.

15. Rampersaud YR, Pik JHT, Salonen D, Farooq S. Clinical Accuracy of Fluoroscopic Computer-Assisted Pedicle Screw Fixation: A CT Analysis. Spine 30( 7): E183-E190, April 2005. 
16. Ughwanogho E, Patel NM, Baldwin KD, Sampson NR, Flynn JM. Computed Tomography-Guided Navigation of Thoracic Pedicle Screws for Adolescent Idiopathic Scoliosis Results in More Accurate Placement and Less Screw Removal. Spine 37(8):E473-E478, April 2012.

17. Tan SB. Clinical and radiological outcomes of minimally invasive versus open transforaminal lumbar interbody fusion. Spine 34(13):1385-9, June 2009.

18. Smith ZA, Fessler RG. Paradigm changes in spine surgery - evolution of minimally invasive techniques. Nature Reviews - Neurology 8(8):443-450, August 2012.

19. Liu J. Comparison of one-level minimally invasive and open transforaminal lumbar interbody fusion in degenerative and isthmic spondylolisthesis grades 1 and 2. European Spine Journal 19(10):1780-4, 2010.

20. Abdullah KG, Bishop FS, Lubelski D, Steinmetz MP, Benzel EC, Mroz TE. Radiation Exposure to the Spine Surgeon in Lumbar and Thoracolumbar Fusions With the Use of an Intraoperative Computed Tomographic 3-Dimensional Imaging System. Spine 37(17):E1074-E1078, August 2012.

21. Kim WK. Use of navigation-assisted fluoroscopy to decrease radiation exposure during minimally invasive spine surgery. Spine Journal 8(4):584-90, July-August 2008.

22. Learch TJ, Massie JB, Pathria MN, Ahlgren BA, Garfin SR. Assessment of Pedicle Screw Placement Utilizing Conventional Radiography and Computed Tomography: A Proposed Systematic Approach to Improve Accuracy of Interpretation. Spine 29(7):767-773, April 2004.

23. Medtronic Inc. O-arm Complete Multidimensional Surgical Imaging System. Dosimetry Report 3D and HD3D Models. Document Nr: BI-700-00027-041 Rev: 1, 20 March 2008.

24. Mannion RJ. A comparison of CT-based navigation techniques for minimally invasive lumbar pedicle screw placement. Journal of Spinal Disorders and Techniques 24(1):E1-5, February 2011.

25. Sanborn MR, Thawani JP, Whitmore RG, Shmulevich M, Hardy B, Benedetto C, Malhotra NR, Marcotte P, Welch WC, Dante S, Stein SC. Cost effectiveness of confirmatory techniques for placement of pedicle screws. Neurosurgical Focus 33 (1):E12, July 2012.

26. Schizas C, Michel J, Kosmopoulos V, Theumann N. Computer tomography assessment of pedicle screw insertion in percutaneous posterior transpedicular stabilization. European Spine Journal 16:613-617, 2007.

27. Smith ZA, Sugimoto K, Lawton CD, Fessler RG. Incidence of Lumbar Spine Pedicle Breach Following Percutaneous Screw Fixation: A Radiographic Evaluation of601 Screws in 151 Patients. Journal of Spinal Disorders and Techniques. 2012 Jun 7. [Epub ahead of print].

28. Bose B, Wierzbowski LR, Sestokas AK. Neurophysiologic Monitoring of Spinal Nerve Root Function During Instrumented Posterior Lumbar Spine Surgery. Spine 27(13):1444-1450, July 2002.

29. Raynor BL, Lenke LG, Bridwell KH, Taylor BA, Padberg AM. Correlation Between Low Triggered Electromyographic Thresholds and Lumbar Pedicle Screw Malposition. Analysis of 4857 Screws. Spine 32(24):2673-2678, November 2007.

30. Raynor BL, Lenke, LG, Bridwell, KH, Taylor, BA, Padberg, AM. Correlation Between Low Triggered Electromyographic Thresholds and Lumbar Pedicle Screw Malposition - Analysis of 4857 Screws. Spine 32(24):2673-2678, November 2007. 
31. Kantelhardt SR, Martinez R, Baerwinkel S, Burger R, Giese A, Rohde V.

Perioperative course and accuracy of screw positioning in conventional, open roboticguided and percutaneous robotic-guided, pedicle screw placement. European Spine Journal 20:860-868, 2011.

32. Cahill KS, Wang MY. Evaluating the Accuracy of Robotic Assistance in Spine Surgery. Neurosurgery 71(2):N20-N21, August 2012.

33. Ringel F, Stüer C, Reinke A, Preuss A, Michael Behr M, Auer F, Stoffel M, Meyer B. Accuracy of Robot-Assisted Placement of Lumbar and Sacral Pedicle Screws. A Prospective Randomized Comparison to Conventional Freehand Screw Implantation. Spine 37(8):E496-E501, April 2012.

\section{Corresponding Author}

Martin Wood, Brisbane Clinical Neuroscience Centre, Mater Private Clinic, South Brisbane, Qld 4101 Australia.

\section{Disclosures}

The authors declare no financial disclosures.

Copyright (C) 2014 ISASS - International Society for the Advancement of Spine Surgery. To see more or order reprints or permissions, see http://ijssurgery.com. 